\title{
Septic Shock Secondary to Salmonella Enterica Ssp. Arizonae in an Immunocompetent Albanian Male
}

\author{
Rapose $\mathrm{A}^{* 1}$ and Dudiki $\mathrm{N}^{2}$ \\ ${ }^{1}$ Division of Infectious Diseases, Reliant Medical Group, Massachusetts, USA \\ ${ }^{2}$ Division of Internal Medicine, Saint Vincent Hospital, Massachusetts, USA
}

${ }^{*}$ Corresponding author: Rapose A, MD, FACP, Division of Infectious Diseases, Reliant Medical Group, 123 Summer Street, Suite 220, Worcester, Massachusetts, USA 01608, Fax: 5083683123, Tel: 5083683122, E-mail: alwyn.rapose@reliantmedicalgroup.org

Citation: Rapose A, Dudiki N (2016) Septic Shock Secondary to Salmonella Enterica Ssp. Arizonae in an Immunocompetent Albanian Male. J Case Rep Stud 4(3): 301. doi: 10.15744/2348-9820.4.301

Received Date: April 03, 2016 Accepted Date: June 17, 2016 Published Date: June 20, 2016

\begin{abstract}
Salmonella-associated diarrhea is a common cause of community-acquired gastroenteritis. Some species of salmonella are associated with invasive diseases like meningitis, endocarditis and septicemia. A 75-year-old Albanian male presented to the emergency department in septic shock. He had history of severe watery diarrhea and high fever of 5 days duration. His stool had a dark green watery consistency imparting a "green pea soup" appearance. Emergency laboratory work-up revealed leukocytosis and acute renal injury. Blood cultures as well as stools yielded S. enterica ssp. arizonae. He was treated with intravenous antibiotics and aggressive fluid resuscitation. Detailed questioning failed to reveal the usual reported risk factors for invasive salmonella infection.
\end{abstract}

Keywords: Salmonella; Subspecies arizonae; Septic shock; Diarrhea

\section{Introduction}

Salmonella enterica ssp. arizonae are Gram-negative bacilli and members of the Enterobacteriaceae family. They are most commonly found in reptiles especially snakes [1]. Cases have also been reported in poultry, pork [2], and rattle snake-basedfolk medicines $[3,4]$. The spectrum of manifestations associated with $S$. enterica ssp. arizonae infection range from benign or severe gastroenteritis [5,6] to severe infections like complicated urinary tract infections [7], septic arthritis and osteomyelitis [8,9], endocarditis [10], meningitis [11] and septicemia [5,8]. The majority of the infections caused by S. enterica ssp. arizonae occur in immunocompromised patients [5,10]. Our case is unique in that the patient neither had known exposure to reptiles or contaminated foods, nor had any known immuno-compromising condition. In addition, this is the first case report of infection with this species of salmonella in the state of Massachusetts, USA.

\section{Case presentation}

A 75-year-old male presented to the emergency department in septic shock. He had history of severe watery diarrhoea and high grade fever of five days duration. He had a significant past medical history of hypertension, enlarged prostate and cigarette smoking for over 50 years. He was originally from Albania, visiting his family in America, arrived one month prior to onset of symptoms. He did not give any history of sick contacts, recent intake of food from restaurants or recent antibiotic use. He denied any overthe-counter medications, food supplements or any unusual hobbies or activities. He owned no pet animals or birds and denied any contact with wild animals. Clinical examination revealed a temperature of $102.4{ }^{\circ} \mathrm{F}$, blood pressure ranging from $80-90 \mathrm{~mm}$ Hg systolic over 50-60 mm Hg diastolic, with pulse 120/min, respiratory rate $24 / \mathrm{min}$. His abdomen was soft and non-tender. A rectal tube was placed secondary to severe watery diarrhoea. His stool had a striking dark green watery appearance (Figure 1), with green-pea-soup appearance in the faecal collecting system (Figure 2).

Laboratory investigations revealed leucocytosis with bandemia ( $13 \times 10^{-9} / 1,61 \%$ bands). He also had acute renal injury with creatinine of $2.71 \mathrm{mg} / \mathrm{dl}$. Serum lactic acid was $3.9 \mathrm{mmol} / \mathrm{l}$. Blood cultures (BacT/Alert 3D, bioMerieux) returned positive four out of four bottles: gram negative rod in less than twenty four hours. Final identification was Salmonella enterica ssp. arizonae which was sensitive to ampicillin, ciprofloxacin and trimethoprim/sulfamethoxazole (Massachusetts State Laboratory). Stool cultures revealed the same organism. Stools were negative for shigella, campylobacter, enterohemorrhagic E.coli and Clostridium difficile toxin. Examination for ova and parasites was also negative. Urine examination revealed moderate red blood cells (10-50/hpf), rare squamous epithelial cells (0-1/lpf) and few bacteria (1-29/hpf), urine culture was negative. Chest radiography revealed patchy 
airspace disease within the medial right lung base and also in the retro-cardiac left base region. Computed tomography (CT) of abdomen and pelvis revealed circumferential wall thickening of the distal ileal loops, terminal ileum, and cecum to the splenic flexure as well as the sigmoid colon with sparing of the descending colon and rectum. HIV test was negative.

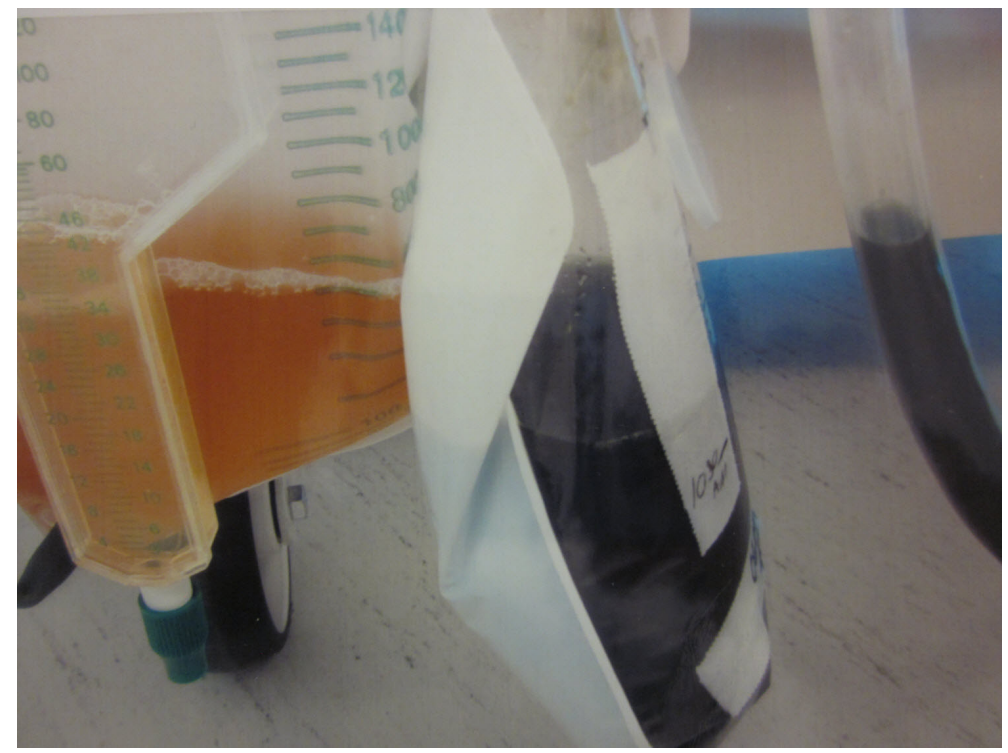

Figure 1: Dark green to black liquid stools

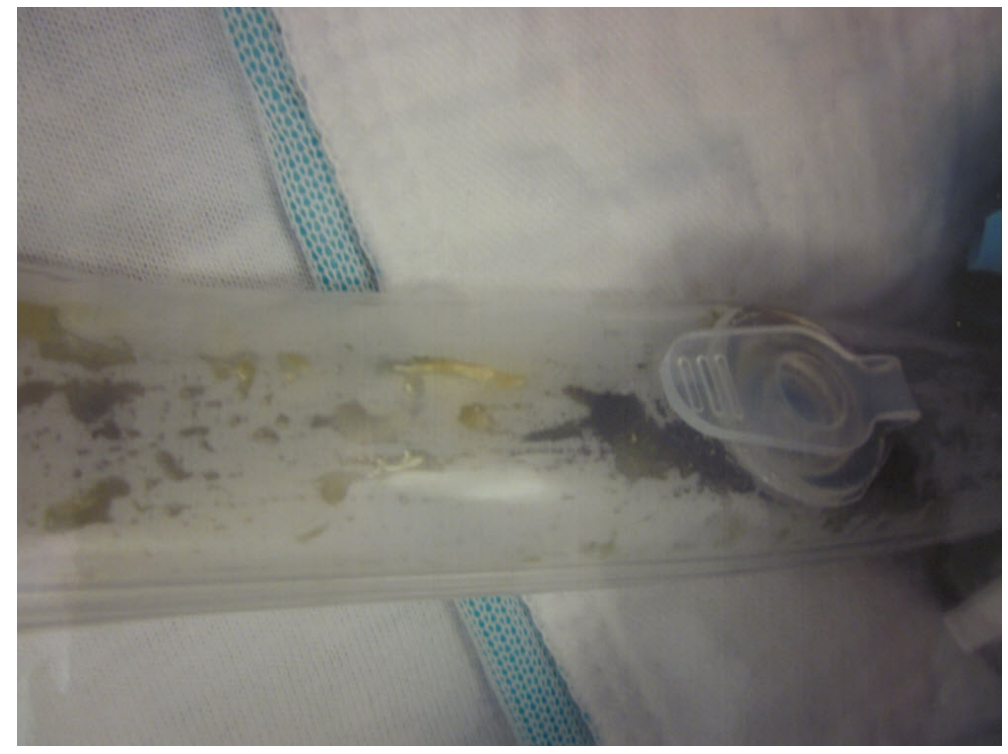

Figure 2: Green-pea-soup coloured stool in the faecal collecting tube

The patient was initially treated empirically with ceftriaxone $2 \mathrm{~g}$ IV daily along with metronidazole 500 mg IV every eight hours. The patient required fluid resuscitation and pressor support with phenylephrine for three days. Once cultures identified the organism and sensitivities were available, antibiotics were changed to ciprofloxacin $400 \mathrm{mg}$ IV twice daily, then to oral ciprofloxacin $500 \mathrm{mg}$ twice a day to complete the course. He had slow clinical improvement and became afebrile after one week, with normalization of the leucocyte count, creatinine and lactic acid. Follow-up blood cultures ( 2 sets) done in the hospital 5 days after admission were negative. The patient received a total of 14 days of antibiotics. At follow-up 1 week after completion of antibiotic course he remained well, free of diarrhoea and fever. A follow-up telephone call one week after that also confirmed complete recovery with no clinical relapse. Unfortunately follow-up stool cultures could not be obtained due to lack of insurance coverage and patient was not in favor of paying out-of-pocket for proof-of-cure follow-up tests after his clinical symptoms had resolved.

\section{Discussion}

S. enterica ssp. arizonae is a gram-negative bacillus and a member of the enterobacteriaceae family. It is most commonly found in cold blooded reptiles especially snakes which act as reservoirs [1], but there have been reported cases of infection in poultry, pork [2], and from ingestion of rattle snake-based folk-medicines [3,4]. When we reviewed the medical literature, we noted that infection with this species of salmonella was rare, associated with exposure to reptiles or ingestion of snake based traditional 
remedies in the majority of cases and in a minority of cases - to the ingestion of eggs and meat contaminated with the bacterium. Mahajan and colleagues reported a case of fatal gastroenteritis with this organism [6]. In their review of 17 other human cases, there was consistent history of contact with reptiles. However, source of infection may not always be easily identified. Di Bella and colleagues [5] reported their case presenting with severe diarrhoea but there was no history of contact with reptiles. They questioned whether his infection could have been caused by ingestion of contaminated food like Italian pasta or cheese. They also reviewed infections reported from Europe. Exposure to snakes and other reptiles was the most common reported risk factor; "travel" was a risk factor in a majority of other case reports. In a review of 23 cases with osteoarticular infection, Schneider and colleagues [9] were able to identify source of infection in only 12 patients. In our case, a detailed inquiry from the patient and his family about possible contact with reptiles or intake of contaminated meat or eggs, either in Albania or the United States was unrevealing.

The spectrum of infections caused by S. enterica ssp. arizonae ranges from mild to severe gastroenteritis to severe infections like complicated urinary tract infections [7], osteomyelitis [8,9], endocarditis [10], meningitis [11] and septicemia [5,8]. Our patient presented with severe diarrhoea, acute renal injury and septic shock requiring fluid resuscitation and pressor support. Review of literature reveals that the majority of infections caused by $S$. enterica ssp. arizonae occur in immunocompromised patients $[5,8,10]$ with malignancy, HIV infection, organ transplantation or other systemic diseases requiring immunosuppressive therapy. Severe infection may be fatal - four out of the 23 patients in one study [9] died. Infections in neonates and infants can be fatal [6,11] as they do not have a mature immune system. PCR can be used to screen stool samples for detection of etiology for bacterial diarrhea and has been shown to reduce turn-around-time as well as increase detection rates [12]. Multiple PCR techniques have also been used to identify specific salmonella species as part of investigations in an outbreak [13]. Unfortunately PCR testing was not available at our hospital and sample was sent to the state laboratory for final identification. Ours is the first case of S. enterica ssp. arizonae infection reported from the state of Massachusetts, USA. Also, he did not have the previously described immunecompromising risk factors for this rare infection. Unfortunately detailed laboratory work-up after discharge from hospital was not possible because patient had no insurance coverage as he was visiting from Albania and was unable to pay for further testing. Thus work-up for immunoglobulin levels and other immune deficiencies could not be achieved. However, his age and lack of history of recurrent infections in the past was considered a surrogate marker for adequate immune status prior to this event. The mode of acquiring this infection - whether it was acquired in the USA or from Albania - could not be determined. Surveillance for similar cases is important to identify some as yet unknown risk factor in otherwise healthy individuals as well as some heretheto unknown source of infection. There may also be an increased incidence of infection with S. enterica spp. arizonae as it becomes popular to keep snakes and other exotic reptiles as pets [8]. Mortality from severe infection has been described, but the patient described in our report was successfully treated, with complete resolution of the infection which implies good response to antibiotics chosen based on sensitivity report.

\section{References}

1. Grupka LM, Ramsay EC, Bemis DA (2006) Salmonella surveillance in a collection of rattlesnakes (Crotalus spp.). J Zoo Wildl Med 37: 306-12.

2. Evangelopoulou G, Kritas S, Govaris A, Burriel AR (2014) Pork meat as a potential source of Salmonella enterica subsp. arizonae infection in humans. J Clin Microbiol 52: 741-4.

3. Cone LA, Boughton WH, Cone LA, Lehv LH (1990) Rattlesnake capsule-induced Salmonella arizonae bacteremia. West J Med 153: 315-6.

4. Kraus A, Guerra-Bautista G, Alarcón-Segovia D (1991) Salmonella arizona arthritis and septicemia associated with rattlesnake ingestion by patients with connective tissue diseases. A dangerous complication of folk medicine. J Rheumatol 18: 1328-31.

5. Di Bella S, Capone A, Bordi E, Johnson E, Musso M, et al. (2011) Salmonella enterica ssp. arizonae infection in a 43-year-old Italian man with hypoglobulinemia: a case report and review of the literature. J Med Case Rep 5: 323.

6. Mahajan RK, Khan SA, Chandel DS, Kumar N, Hans C, et al. (2003) Fatal case of Salmonella enterica subsp. arizonae gastroenteritis in an infant with microcephaly. Clin Microbiol 41: 5830-2.

7. Landron C, Le Moal G, Roblot F, Grignon B, Becq-Giraudon B (2003) Arizonae subspecies Salmonella enterica urinary infection with confusional syndrome. Presse Med 32: 696-7.

8. Kolker S, Itsekzon T, Yinnon AM, Lachish T (2012) Osteomyelitis due to Salmonella enterica subsp. arizonae: the price of exotic pets. Clin Microbiol Infect 18: $167-70$.

9. Schneider L, Ehlinger M, Stanchina C, Giacomelli MC, Gicquel P, et al. (2009) Salmonella enterica subsp. arizonae bone and joints sepsis. A case report and literature review. Orthop Traumatol Surg Res 95: 237-42.

10. Starakis I, Siagris D, Karatza C, Solomou H, Bassaris H (2007) Endocarditis due to Salmonella enterica subsp. arizonae in a patient with sickle cell disease: a case report and review of the literature. Cardiovasc Hematol Disord Drug Targets 7: 199-204.

11. Lakew W, Girma A, Triche E (2013) Salmonella enterica Serotype Arizonae Meningitis in a Neonate. Case Rep Pediatr doi: $10.1155 / 2013 / 813495$.

12. Van Lint P, De Witte E, Ursi JP, Van Herendael B, Van Schaeren J (2016) A screening algorithm for diagnosing bacterial gastroenteritis by real-time PCR in combination with guided culture. Diagn Microbiol Infect Dis S0732-8893(16)30057-8.

13. Hashemi A, Baghbani-Arani F (2015) The effective differentiation of Salmonella isolates using four PCR-based typing methods. J Appl Microbiol 118: 1530-40. 


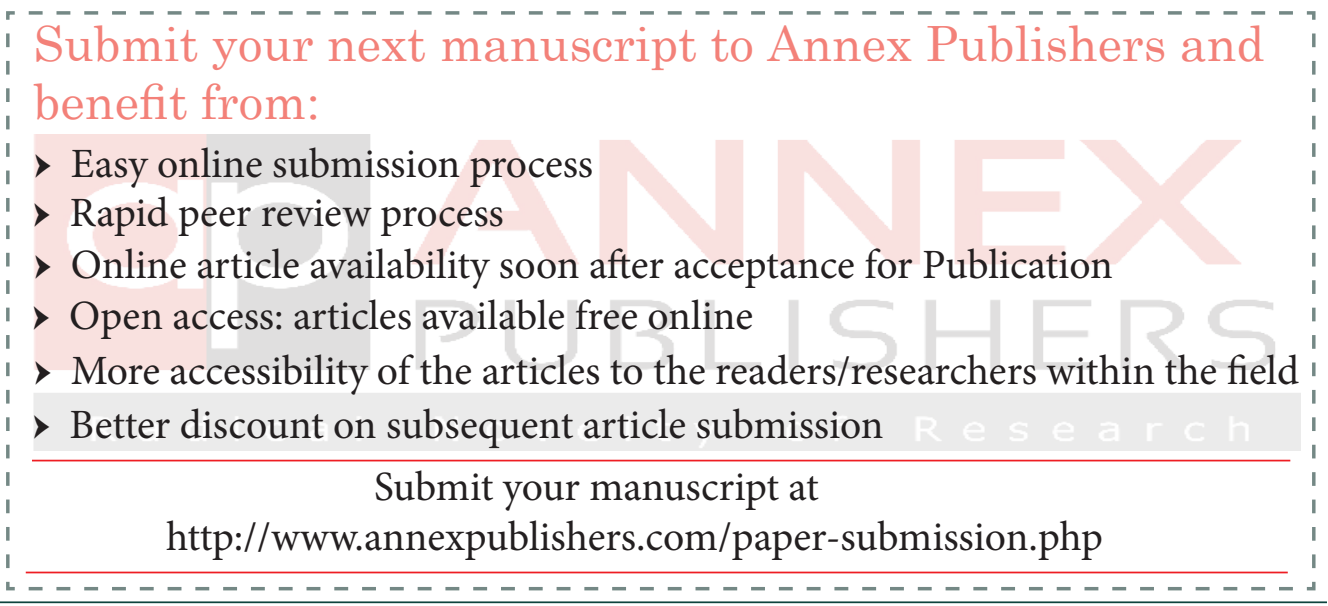

